Groman, N. B. \& Memmer, R. (1958). J. gen. Microbiol. 19, 634-644

\title{
Lysogeny and Conversion in Mitis and Mitis-Like Corynebacterium diphtheriae
}

\author{
BY N. B. GROMAN AND RAMONA MEMMER \\ Department of Microbiology, School of Medicine, The University of Washington, \\ Seattle, Washington, U.S.A.
}

SUMMARY: Fifty-six toxigenic mitis and mitis-like Corynebacterium diphtheriae strains isolated in the western part of the United States and Canada were examined for lysogeny and for the relationship of their phages to conversion to toxigenicity. Twenty-five strains $(46 \%)$ were demonstrably lysogenic. Twenty-two of the phages from the toxigenic strains were able to convert a sensitive non-toxigenic strain to toxigenicity. Due to technical limitations three phages could not be tested in a similar manner. On the basis of serological relationships, cross-immunizing ability and host range all 22 converting phages proved to be very closely related. They were also related to $\beta$ - and $\gamma$-phages previously studied in connexion with the phenomenon of conversion to toxigenicity by bacteriophage.

Freeman's discovery (1951) that non-toxigenic Corynebacterium diphtheriae were converted to toxigenicity by bacteriophage was recognized immediately as an observation of great importance to a broadened understanding of the epidemiology of diphtheria. In spite of this, little information has been published relating directly to the natural occurrence or significance of the phenomenon he described. In the present study some of the questions which have been raised in relation to epidemiology have been investigated. A group of toxigenic mitis and mitis-like $C$. diphtheriae isolated in western United States and Canada were studied with the following questions in mind. How many of the isolates are lysogenic? How many of the lysogenic strains carry a phage capable of converting a sensitive non-toxigenic strain to toxigenicity? Are the phages carried by these strains related to each other and/or to the $\beta$ - and $\gamma^{\prime}$-converting phages (Groman \& Eaton, 1955) previously studied in association with mitis-like $C$. diphtheriae?

\section{MATERIALS AND METHODS}

Bacteriophage and bacterial strains. Lyophilized cultures from the stock collection of Corynebacterium diphtheriae strains at the University of Washington were used. The cultures had been collected by Dr V. J. Freeman. Stock cultures of these strains were maintained on slopes of trypticase soy medium (Baltimore Biol. Labs.), supplemented with $1 \%$ yeast extract and were stored at $4^{\circ}$. Transfers were generally made every 5-6 weeks. A list of the strains arranged according to their geographical origin is given in Table 1. Information was not available concerning the origin of each culture; therefore, it is possible that some of these cultures represent multiple isolates from the same individual. A number of other bacterial strains, C4, C4Sr (a streptomycin 
resistant mutant of $\left.\mathrm{C}_{4}\right), \mathrm{C} 4 / \beta, \mathrm{C} 4(\beta), \mathrm{C} 4(\gamma), \mathrm{C} 7$ and $\mathrm{C} 1180$ were employed as phage indicators during this investigation and represent strains which have been studied in relation to the conversion phenomenon. These strains were maintained on Loeffler's medium.

Table 1. Strains of toxigenic Corynebacterium diphtheriae examined in this study

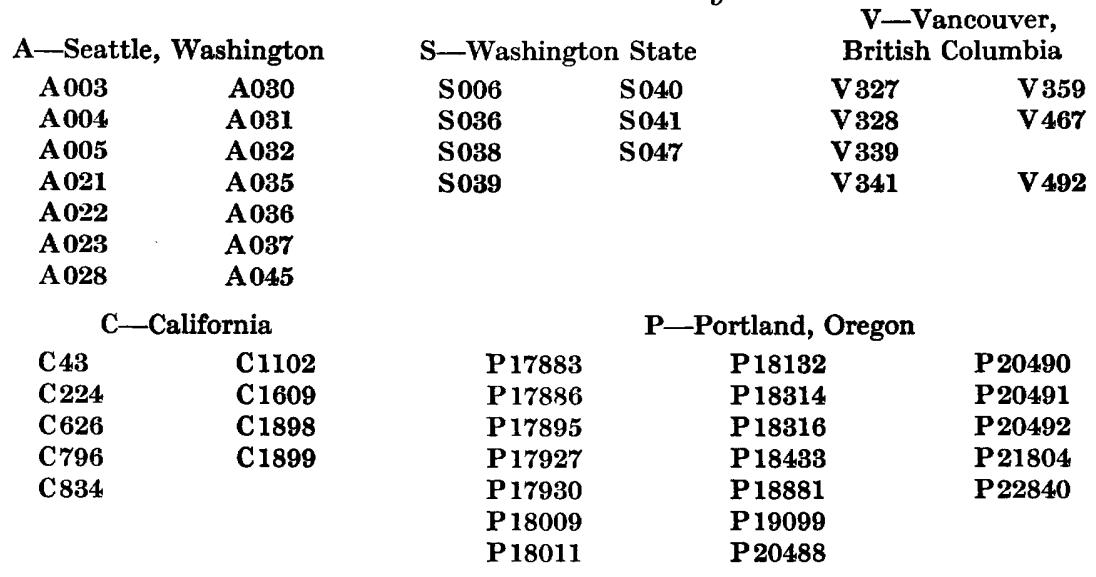

In addition to the phages isolated during this study, certain phages previously studied in this laboratory were employed. Phages $\beta$ and $\gamma$ are related temperate phages; $\beta$ is able to convert sensitive non-toxigenic strains of Corynebacterium diphtheriae to toxigenicity, while $\gamma$ cannot. Phages $\beta^{\prime}$ and $\gamma^{\prime}$ are recombinants of $\beta$ and $\gamma$. Phage $B$ is a virulent mutant of $\beta$; phage $B h$ is a host range mutant of $B$. The critical relationships among stock phages, stock strains, and the toxigenicity of stock strains are given in Table 2.

Table 2. The interrelationships of Corynebacterium diphtheriae strains and phages

\begin{tabular}{|c|c|c|c|c|c|c|c|c|}
\hline \multirow[b]{2}{*}{ Strains } & \multicolumn{4}{|c|}{ Sensitivity to phage } & \multicolumn{3}{|c|}{$\begin{array}{l}\text { Tests for lysogeny on } \\
\text { indicator strains }\end{array}$} & \multirow[b]{2}{*}{$\begin{array}{l}\text { Toxi- } \\
\text { genicity }\end{array}$} \\
\hline & $\begin{array}{c}\beta \\
\text { and } \\
\beta^{\prime}\end{array}$ & $\begin{array}{c}\gamma \\
\text { and } \\
\gamma^{\prime}\end{array}$ & $\mathbf{B}$ & Bh & $\begin{array}{c}\mathrm{C} 4(\beta) \\
\text { and } \\
\mathrm{C} 4\left(\beta^{\prime}\right)\end{array}$ & $\mathrm{C} 4 / \beta$ & $\begin{array}{c}\mathrm{C} 4(\gamma) \\
\text { and } \\
\mathrm{C} 4\left(\gamma^{\prime}\right)\end{array}$ & \\
\hline $\mathrm{C}_{4}$ & + & + & + & + & - & - & - & - \\
\hline $\mathrm{C} 4(\gamma)$ & + & - & + & + & + & + & - & - \\
\hline $\mathrm{CA}\left(\gamma^{\prime}\right)$ & + & - & + & + & + & + & - & + \\
\hline $\mathbf{C} 4(\beta)$ & - & + & - & + & - & - & + & + \\
\hline$C 4\left(\beta^{\prime}\right)$ & - & + & - & + & - & - & + & - \\
\hline $\mathrm{C} 4 / \beta$ & - & + & + & + & - & - & - & - \\
\hline
\end{tabular}

Bacteriological methods. Difco heart-infusion broth (HIB) and a Proteose peptone salt (PPS) medium were used throughout the work for the propagation of the bacterial strains and bacteriophage. The heart-infusion medium was used exclusively for dilution and for plaque counting and had a final 
pH of $\mathbf{7 \cdot 2 - 7 \cdot 4}$. The PPS medium, which consisted of $2 \%$ Proteose peptone (Difco) and $0.5 \%$ sodium chloride at a final $\mathrm{pH}$ of $7 \cdot 8$, was also employed in the 'in vitro' test for toxigenicity. For this test the salt concentration was reduced to $0.25 \%$ and the medium was supplemented with $20 \%$ sheep serum. Toxin production was also determined by the intracutaneous method in guinea-pigs.

Both HIB and Neill's broth base medium were used in determining sugar fermentations. Neill's broth base consisted of $1 \%$ beef extract, $1 \%$ Proteose peptone no. 3 (Difco), and $0.5 \% \mathrm{NaCl}$ and had a final $\mathrm{pH}$ of 7.3. Bromcresol purple was used as the indicator. Sucrose and glucose were incorporated into the base medium prior to sterilization, while maltose, starch and glycogen were added aseptically subsequent to sterilization. Final sugar concentrations were those recommended in Diagnostic Procedures and Reagents (1950). Inocula consisted of $0.1 \mathrm{ml}$. of overnight cultures grown in the particular base medium to be used. Reactions were recorded at 24,48 and $72 \mathrm{hr}$. and at 1 week.

To test for haemolysin production, $1.0 \mathrm{ml}$. of a $48 \mathrm{hr}$. HIB culture of the bacterium was added to $1.0 \mathrm{ml}$. of a $2 \%$ suspension of fresh human red blood cells which had been washed three times in 0.01 M-phosphate buffered saline, $\mathrm{pH} \mathrm{7 \cdot 0.}$. The tubes were placed in $\mathrm{a} 37^{\circ}$ water bath for $1 \mathrm{hr}$., then refrigerated overnight. Haemolysis was read as present or absent. A control tube of sterile broth plus the red blood cells was included and was always negative.

Nitrate reduction was tested by incorporating $0 \cdot 1 \% \mathrm{KNO}_{3}$ into HIB. Onetenth ml. of an overnight HIB culture was used as the inoculum. Reduction to nitrite was tested at $48 \mathrm{hr}$. Cultures negative at this time were retested at the end of a 1 week incubation period, and those still negative were tested for the presence of nitrate by adding a few grains of powdered zinc to the medium following the addition of $0.5 \mathrm{ml}$. each of $0.8 \%$ sulphanilic acid and $0.5 \%$ alphanaphthylamine.

Christensen's urea agar slants (1946) adjusted to $\mathrm{pH} 7 \cdot 2$, were used to test for urease production. The slants were streaked and stabbed. Reactions were checked at 24, 48 and $72 \mathrm{hr}$. and at 1 week.

Mueller's serum tellurite medium (Mueller \& Miller, 1946) was used to determine colonial morphology.

Bacteriophage technique. Plaque counts, tests for phage sensitivity and for lysogeny, and preparation of stocks of phages $\mathrm{B}, \mathrm{Bh}, \beta$ and $\gamma$ were carried out as previously outlined (Groman \& Eaton, 1955). Stocks of phages from newly identified lysogenic strains were prepared in a manner similar to that described by Parsons (1955).

The isolation of lysogenized $\mathrm{C} 4 \mathrm{Sr}$ strains. In the course of the present work strain $\mathrm{C} 4 \mathrm{Sr}$ was lysogenized with 22 phages derived from newly identified lysogenic strains. $\mathrm{C} 4 \mathrm{Sr}$ was employed to provide a marker (streptomycin resistance) which would distinguish the recipient strain from the phage donor strains, which were all streptomycin sensitive. In each case strain $\mathrm{C} 4 \mathrm{Sr}$ was exposed to the phage either in broth or in an agar overlay, and following lysis and regrowth the culture or material from a plaque was streaked out on heart- 
infusion agar. Well-isolated clones were then tested for phage production. One productive clone was restreaked and five well-isolated subclones retested for phage production. When necessary, the re-isolation process was continued until all subclones produced phage. At this time a subclone was transferred to stock as a lysogenic derivative of strain $\mathrm{C} 4 \mathrm{Sr}$. All cultures were checked and found resistant to streptomycin; and in addition, all were shown to agglutinate with an antiserum to $\mathrm{C} 4 \mathrm{Sr}$. The latter test provided a double check on the relationship of most of the lysogenic strains to the original $\mathrm{C} 4 \mathrm{Sr}$ strain, since 19 of the 22 donor strains were antigenically unrelated to $\mathrm{C}_{4} \mathrm{Sr}$.

\section{RESULTS}

Characterization of the strains. In selecting strains of Corynebacterium diphtheriae for study, those catalogued as mitis type were chosen. Since there were no experimental records of the criteria employed in typing these strains a redetermination of type was undertaken along with certain other biochemical tests.

All the strains which are listed in Table 1 produced acid from glucose and maltose, did not ferment sucrose, glycogen or starch, did not produce urease and with the exception of SO 47 reduced nitrate to nitrite. None produced a pellicle when grown in broth. All the strains listed were toxigenic. Their cellular morphology was in agreement with that expected for Corynebacterium diphtheriae. Haemolysin production and colonial morphology were more variable among these strains. Six strains (V 327, V 328, V329, V341, P18314, P 20490, failed to produce haemolysin and 8 strains (P 17895, P 17930, P 18011, P18132, P18316, P19099, P20491, P21804) gave both positive and negative results in a series of three tests.

The typical colonial morphology of mitis strains described by Mueller \& Miller (1946) on serum tellurite medium, i.e. colonies 1-1.5 mm., black, convex and glistening, was exhibited by only a few of the strains tested. The majority were atypical in that their colonies exhibited black centres with a grey-white edge or black centres with a lighter concentric ring and an outer white edge. A few had colonies with grey or brown centres with a white edge. In spite of these differences the large size, convex contour, glistening appearance, and black or dark centres clearly qualified these colonies as mitis or mitis-like rather than gravis or intermedius. On the basis of all these observations the 56 strains studied in this investigation were classified as mitis or mitis-like Corynebacterium diphtheriae.

The extent of lysogeny. Two methods were employed in testing for lysogeny. In the first method supernatant fluids of the cultures to be tested were pooled in groups of three. Each culture was grown to approximately $4 \times 10^{8} \mathrm{cell} / \mathrm{ml}$. and centrifuged for $20 \mathrm{~min}$. at $3000 \mathrm{rev} . / \mathrm{min}$.; equal samples of the supernatants were pooled. A loopful of each pool was spotted on each of the 56 strains being studied and on stock strains $\mathrm{C} 4, \mathrm{C} 7$ and $\mathrm{C} 1180$. When employed as an indicator, each strain was incorporated into an agar overlay as for plaque counting, two plates per indicator strain sufficing for the nineteen pool tests. 
When a given pool exhibited activity, the strains contributing to the pool were then tested individually against the sensitive indicator to determine the phage carrier(s).

In the second method employed a loopful of the supernatant fluid of each culture was spotted on stock strains $\mathrm{C} 4$ and $\mathrm{C} 7$. These indicators were selected on the basis of their known sensitivity to stock Corynebacterium phages. On the basis of the pool tests it was apparent that strain $\mathbf{A 0 2 8}$ displayed a wider range and a greater sensitivity to phage than our stock strains, and each toxigenic strain was tested individually on it as well. One other stock strain, C1180, was also sensitive to a number of phages. However, not all strains were tested on it individually as it appeared to be sensitive to the phages active on $\mathrm{C} 4$ and $\mathrm{C} 7$ and in fact was a second isolate from the same diphtheria patient yielding C7 (Freeman, 1951).

The results of tests with all identifiable lysogenic strains and three nonlysogenic strains are given in Table 3 . The host range of the phages carried by the lysogenic strains is given in columns 2 to 8 . The indicator strains

Table 3. The sensitivity of Corynebacterium diphtheriae strains to stock phage and the host range of their carried phages

\begin{tabular}{|c|c|c|c|c|c|c|c|c|c|c|c|}
\hline \multirow[b]{2}{*}{ Strains } & \multicolumn{7}{|c|}{ Tests for lysogeny on indicator strains } & \multicolumn{4}{|c|}{ Sensitivity to phage } \\
\hline & A 028 & C1180 & C4 & $\mathrm{C} 7$ & $\mathrm{C} 4(\beta)$ & $\mathrm{C}_{4} / \beta$ & C4 $4(\gamma)$ & $\gamma$ & $\beta$ & $\mathbf{B}$ & $\mathbf{B h}$ \\
\hline A023 & + & + & + & + & + & + & - & - & + & + & + \\
\hline C1899 & + & + & + & + & + & + & - & - & + & + & + \\
\hline $\mathrm{C} 1102$ & + & + & + & + & + & + & - & - & + & + & - \\
\hline $\mathrm{C} 43$ & + & + & + & + & + & + & - & - & - & + & + \\
\hline C1898 & + & + & + & + & + & - & - & - & - & + & + \\
\hline A003 & + & + & + & + & + & + & - & - & - & + & + \\
\hline A004 & + & + & + & + & + & + & - & - & - & + & + \\
\hline A005 & + & + & + & + & + & + & - & - & - & + & + \\
\hline A022 & + & + & + & + & + & + & - & - & - & + & + \\
\hline So06 & + & + & + & + & + & + & - & - & - & + & + \\
\hline S036 & + & + & + & + & + & + & - & - & - & + & + \\
\hline S038 & + & + & + & + & + & + & - & - & - & + & + \\
\hline S 039 & + & + & + & + & + & + & - & - & - & + & + \\
\hline S040 & + & + & + & + & + & + & - & - & - & + & + \\
\hline C224 & + & + & + & + & + & + & - & - & - & + & - \\
\hline V 467 & + & + & + & + & + & + & - & - & - & + & - \\
\hline C796 & + & + & + & + & + & + & - & - & - & - & - \\
\hline C834 & + & + & + & + & + & + & - & - & - & - & - \\
\hline $\mathrm{C} 1609$ & + & + & + & + & + & + & - & - & - & - & - \\
\hline A030 & + & + & + & + & + & + & - & - & - & - & - \\
\hline A031 & + & + & + & + & + & + & - & - & - & - & - \\
\hline A037 & + & + & + & + & + & + & - & - & - & - & - \\
\hline P17886 & + & + & + & - & - & - & _- & - & - & - & - \\
\hline P18011 & + & - & - & - & - & - & - & - & - & - & - \\
\hline P21804 & + & - & - & - & - & - & - & - & - & - & - \\
\hline V359* & _ & - & - & - & - & - & _ & - & + & + & - \\
\hline A 045 & - & - & - & - & - & - & - & - & + & + & + \\
\hline A028 & - & - & - & - & - & - & - & + & + & + & + \\
\hline
\end{tabular}

* This group of three contains the only non-lysogenic organisms exhibiting sensitivity to stock phages. 


\section{Lysogeny and conversion in $\mathrm{C}$. diphtheriae}

listed are all those on which a positive test was obtained. Strain $\mathrm{C} 4(\gamma)$ was included for purposes of characterizing the host range pattern of the phages. The data show that 25 of the 56 cultures tested, or $46 \%$, were lysogenic. The maximum number of lysogenic strains was detected by A 028, although the sensitivity of strains $\mathbf{C 4}, \mathrm{Cr}$ and $\mathrm{C} 1180$ was almost as good. It is clear that $46 \%$ represents a minimal estimate of lysogeny in this group of cultures, since tests with additional indicator strains or the employment of more sensitive methods might well add to the number of positives.

The host range exhibited by the phages carried by these strains was identical, with the exception of the phages carried by strains C1898, P17886, P18011, and P21804. It is worth noting that only one of the 56 toxigenic strains tested, A 028, was sensitive to any of the newly isolated phages as determined by the pool tests. Nontoxigenic indicator strains $\mathrm{C} 4, \mathrm{C} 7$ and $\mathrm{C} 1180$ were also screened for lysogeny in the pool test, but no phage activity was noted.

In addition to checking all 56 strains for lysogeny, each strain was tested for its sensitivity to stock phages (Table 2, columns 9-12). The strains have been arranged in groups which reflect their pattern of phage sensitivity. Of the 56 strains tested, $19(34 \%)$ were sensitive to one or more stock phages. Sixteen of the 19 sensitive strains were members of the lysogenic group and only 3 (the last group in the table) of the $\mathbf{3 1}$ non-lysogenic strains were sensitive. These patterns of phage sensitivity do not correlate with the general geographical origin of the cultures although more detailed knowledge of their origin might reveal some intra-group correlations.

The relationship of the newly isolated phages to $\beta$-and $\gamma$-phages. The relationship between the phages isolated from the lysogenic strains and $\beta$ - and $\gamma$-phages, which are themselves closely related (Groman \& Eaton, 1955), was determined next. As indicated above, with but four exceptions, the phages carried by the lysogenic strains exhibited an identical host range on all available indicator strains. However, host range is not a good criterion for demonstrating relationships among phages. Two criteria which are acceptable are serological relationship and the ability of temperate phages to immunize a given host to attack by the same phage and in most cases to closely related phages.

In order to determine whether the new phages were related to $\beta$ and $\gamma$ each phage was exposed to an antiserum produced against $\gamma$-phage which neutralized both $\beta$ and $\gamma$ at the same rate. Stocks of all but 3 of the unknown phages were prepared by growth on either $\mathrm{C} 4$ or $\mathbf{A} 028$. Ideally the inactivation rate constant of each phage should have been determined over a series of time intervals as a measure of its relationship to $\beta$ and $\gamma$. However, this approach was technically not feasible and as a quantitative measure of relatedness the percentage inactivation of the unknown phage was compared with that of $\beta$-phage over a single time interval.

The results of these experiments, which are given in Table 4, clearly show that on the basis of the serological criterion all the phages isolated from the lysogenic strains are closely related to $\beta$ and $\gamma$ and inferentially to each other. The phages carried by the three lysogenic $\mathbf{P}$ strains were not included in these tests. 
Table 4. The rate of inactivation of the newly isolated phages and $\beta$-phage by $\beta$-phage antiserum

\begin{tabular}{|c|c|c|c|c|c|}
\hline \multirow[b]{2}{*}{$\begin{array}{l}\text { Phage donor } \\
\text { strain }\end{array}$} & \multicolumn{2}{|c|}{$\%$ inactivation* } & \multirow[b]{2}{*}{$\begin{array}{l}\text { Phage donor } \\
\text { strain }\end{array}$} & \multicolumn{2}{|c|}{$\%$ inactivation* } \\
\hline & $\begin{array}{l}\text { Phage from } \\
\text { donor }\end{array}$ & $\beta$-phage & & $\begin{array}{c}\text { Phage from } \\
\text { donor }\end{array}$ & $\beta$-phage \\
\hline $\mathrm{C} 43$ & 40 & 45 & A 022 & 49 & 31 \\
\hline $\mathrm{C224}$ & 57 & 44 & A023 & 42 & 39 \\
\hline C796 & 69 & 31 & $\mathbf{A 0 3 0}$ & 51 & 40 \\
\hline C 834 & 47 & 39 & A031 & 51 & 31 \\
\hline C 1102 & 73 & 45 & A 037 & 55 & 40 \\
\hline C 1609 & 72 & 40 & S006 & 62 & 44 \\
\hline C 1898 & 65 & 31 & S036 & 74 & 46 \\
\hline C1899 & 46 & 45 & S038 & 36 & 45 \\
\hline A003 & 67 & 58 & S039 & 76 & 58 \\
\hline A 004 & 55 & 45 & $\mathrm{SO} 40$ & 60 & 31 \\
\hline A005 & 65 & 33 & V 467 & 41 & 44 \\
\hline
\end{tabular}

* All phages were incubated for $10 \mathrm{~min}$. at $37^{\circ}$ with a $1: 20$ dilution of antiserum. $\beta$-phage inactivation was determined simultaneously with that of the unknown phage. Normal serum controls were included in each experiment. The $10 \mathrm{~min}$. period was selected after preliminary experiments had indicated that inactivation was still progressing logarithmically at this time.

In order to carry out a significant test for the cross-immunizing ability of the newly isolated phages it was necessary to lysogenize a common bacterial host with each of these phages and isolate the lysogenic derivatives. Strain C4 was selected as the appropriate host since it was sensitive to 23 of the total of 25 phages. Since it was also non-toxigenic, the converting ability of these phages could be assessed simultaneously. The methods employed in the isolation of these lysogenic strains were outlined in the Material and Methods section, and it will be recalled that a streptomycin resistant mutant of $\mathrm{C} 4$, i.e. $\mathrm{C} 4 \mathrm{Sr}$, was employed as the recipient host.

Lysogenic $\mathrm{C} 4 \mathrm{Sr}$ derivatives of 22 of the 23 phages active on this strain were isolated. Numerous efforts were made to obtain a strain lysogenized with the phage from P17886 but all failed. Each of the newly isolated strains, which will be designated $\mathrm{C} 4 \mathrm{Sr}(\mathrm{X})$ strains, where $\mathrm{X}$ represents the strain designation of the phage donor, was tested for sensitivity to the phage carried by each of the other $\mathrm{C}_{4} \operatorname{Sr}(X)$ strains. In addition, the phage carried by each $C_{4} \operatorname{Sr}(X)$ strain was tested for its host range on a group of selected indicators. Finally, each $\mathrm{C}_{4} \mathrm{Sr}(\mathrm{X})$ strain was tested for its sensitivity to stock phages $\beta, \gamma, \mathbf{B}$ and $\mathrm{Bh}$. A summary of the findings is given in Table 5.

The data clearly show that each of the phages isolated during this study immunizes a common host to attack by all the others. Coupled with the serological relationship this finding established the relation of these phages to $\beta$ - and $\gamma$-phage and to each other. With three exceptions this common immunizing property extends to immunizing $\mathrm{C} 4 \mathrm{Sr}$ to stock phages as well. The added resistance of strains $\mathrm{C} 4 \mathrm{Sr}(\mathrm{A}$ 003), $\mathrm{C} 4 \mathrm{Sr}(\mathrm{S038})$ and $\mathrm{C} 4 \mathrm{Sr}(\mathrm{V} 467)$ to $\beta$-phage might suggest that these phages are different in some degree from the others. However, we have observed (unpublished observations) that $\mathrm{C} 4 / \beta$ 
mutants, i.e. mutants resistant to $\beta$-phage, are very common in $\mathrm{C}_{4}$ cultures. It is quite likely that the three exceptions are in fact $\mathrm{C} 4 \operatorname{Sr}(\mathrm{X}) / \beta$ strains and that the phages they carry are similar in all respects to the others.

Table 5. The host range and immunizing ability of phages carried by the $C 4 \operatorname{Sr}(X)$ strains

\begin{tabular}{|c|c|c|c|c|c|c|}
\hline \multirow{3}{*}{$\begin{array}{l}\text { Bacterial } \\
\text { strain }\end{array}$} & \multicolumn{5}{|c|}{ Sensitivity to } & \multirow{3}{*}{$\begin{array}{c}\text { Toxi- } \\
\text { genicity }\end{array}$} \\
\hline & \multirow{2}{*}{$\begin{array}{l}\text { Phages } \\
\text { from } \\
\text { strains } \\
\text { C4Sr(X)* }\end{array}$} & \multicolumn{4}{|c|}{ Stock phage } & \\
\hline & & $\gamma$ & $\beta$ & B & $\mathbf{B h}$ & \\
\hline $\mathrm{C}_{4} \operatorname{Sr}(\mathrm{X}) \dagger$ & - & - & + & + & + & + \\
\hline C4Sr(A003) & - & - & - & + & + & + \\
\hline C4Sr(S038) & - & - & - & + & + & + \\
\hline $\mathrm{C} 4 \mathrm{Sr}(\mathrm{V} 467)$ & - & - & - & + & + & + \\
\hline A 028 & + & + & + & + & + & + \\
\hline $\mathrm{Cr}$ & + & + & + & + & + & - \\
\hline C4 & + & + & + & + & + & - \\
\hline $\mathrm{C} 4(\gamma)$ & - & - & + & + & + & - \\
\hline $\mathrm{C4}(\beta)$ & + & + & - & - & + & + \\
\hline $\mathrm{C} 4 / \beta$ & + & + & - & + & + & - \\
\hline
\end{tabular}

* $\mathbf{X}$ refers to phages obtained from each of the strains in the following list:

$\begin{array}{lllll}\text { C43 } & \text { C } 1609 & \text { A005 } & \text { A031 } & \text { S006 } \\ \text { C224 } & \text { C1898 } & \text { A022 } & \text { A 037 } & \text { S036 } \\ \text { C796 } & \text { C } 1899 & \text { A 023 } & \text { S038 } & \text { S039 } \\ \text { C834 } & \text { A } 003 & \text { A030 } & \text { S040 } & \text { V467 } \\ \text { C1102 } & \text { A 004 } & & & \end{array}$

$\dagger \mathrm{X}$ refers to all strains in the above list less the $\mathbf{3}$ strains listed individually in the Table.

In addition to a common immunizing ability, all 22 phages had identical host ranges when tested against stock indicator strains (bottom portion of Table 5). On the basis of both of these characteristics it can be stated that all the phages are related to or identical with $\gamma$ or $\gamma^{\prime}$ (see Table 2 also). It will be noted that the pattern of phage sensitivity of the $\mathrm{C} 4 \mathrm{Sr}(\mathrm{X})$ derivatives differs in most instances from that exhibited by the parent strains supplying the phage (see Table 3). This is not unexpected, since the broadened resistance to phage infection exhibited by the parent strain may have developed independently, e.g. by mutation or as a result of lysogenization with other phages. It is this very fact which dictated the need for a common host in testing cross-immunizing ability among the phages isolated in this study. It is also worth noting that most of the parent strains also act as $\beta$-resistant strains in a manner analogous to $\mathrm{C} 4 \mathrm{Sr}(\mathrm{A003}), \mathrm{C} 4 \mathrm{Sr}(\mathrm{S} \mathrm{038})$ and $\mathrm{C} 4 \mathrm{Sr}(\mathrm{V} 467)$, indicating that this type of strain arises under natural conditions.

Converting ability of phages isolated from toxigenic strains. One of the primary purposes of this survey was to determine whether phages isolated from toxigenic strains were able to convert non-toxigenic strains to toxigenicity. As indicated in Table 5, the lysogenic $\mathrm{C} 4 \mathrm{Sr}$ derivatives of 22 phages obtained from toxigenic strains were all toxigenic when tested by either the 'in vitro' plate or guinea-pig intracutaneous method. Thus each toxigenic strain which 
could be tested was carrying a converting phage. The phage from one Oregon strain, P17886, was also tested for its converting ability on $\mathrm{C} 4 \mathrm{Sr}$. When cultures containing $\mathrm{C}_{4} \mathrm{Sr}$ and phage $\mathrm{P} 17886$ were streaked on 'in vitro' plates, positive tests for toxin production were consistently obtained, indicating the presence of a converting phage; but as mentioned earlier, a lysogenic $\mathbf{C} 4 \mathbf{S r}$ derivative could not be isolated.

Serological relationship of the toxigenic strains to $C 4$. Another question studied was whether strains sensitive to the same phages as $\mathrm{C4}$, or strains producing phage which attacked $\mathrm{C} 4$, were serologically related to $\mathrm{C} 4$. To test for such a relationship a slide agglutination test was used in which a $\mathrm{C}_{4}$ agglutinating antiserum was employed. The method of testing was that described by Minzel \& Freeman (1950). Eight of the 56 toxigenic strains agglutinated with $\mathrm{C} 4$ antiserum. These were $\mathrm{C626}, \mathrm{C} 796, \mathrm{C} 834, \mathrm{C} 1609$, V327, $\mathrm{V} \mathrm{328,} \mathrm{V} 339$ and V 341. Of the 23 strains carrying phage active on $\mathrm{C} 4,3$ were serologically related to $\mathrm{C} 4(\mathrm{C} 796, \mathrm{C} \mathrm{834}, \mathrm{C} 1609)$; these strains were from the same geographical area as $\mathrm{C} 4$. Although $\mathrm{C}_{4}$ is sensitive to $\beta$-phage, none of the 6 strains sensitive to $\beta$-phage agglutinated with $\mathrm{C}_{4}$ antiserum. On the basis of these results there is no recognizable relationship between phage production or sensitivity to phage and agglutination as determined by the present test.

\section{DISCUSSION}

There seems to be no question that lysogeny is a common property among the various types of Corynebacterium diphtheriae (Keogh, Simmons \& Anderson, 1938; Thibaut \& Frédéricq, 1952, 1956; Hewitt, 1952; Christensen, 1957). While we have found that $46 \%$ of the toxigenic mitis or mitis-like strains were demonstrably lysogenic, the percentage of lysogeny reported by others among all types of $C$. diphtheriae has ranged from zero to values approximating those found in the current study. A true comparison of the extent of lysogeny among the various types of $C$. diphtheriae would be of theoretical interest; however, such a comparison is devoid of meaning, since in any study the detection of lysogeny depends on the fortuitous selection of appropriate indicator strains.

One of the most important questions raised by the conversion phenomenon is that of the role it plays in the natural evolution of toxigenic strains of Corynebacterium diphtheriae. One approach to this question is to examine toxigenic strains for the presence of converting phage. While lysogeny is a common characteristic of toxigenic $C$. diphtheriae, the presence or absence of converting phages in such strains has not been studied extensively. Hewitt, (1954) reported that 5 toxigenic gravis strains yielded converting phages, and Narbutowicz (1955) isolated a converting phage from a toxigenic mitis strain. In the present study converting phage was isolated from 22 toxigenic mitis or mitis-like strains and in fact was demonstrated in every instance where a reasonably convenient means for its demonstration existed. These findings strengthen the hypothesis that conversion is a major mechanism in the production of toxigenic $C$. diphtheriae. While the presence of a converting 
phage in a toxigenic strain does not prove its role in the production of toxin, the inference is strong.

The finding that all of the converting phages isolated in the present study appear to be identical merits mention. One obvious explanation is that the indicator strains employed could only detect phages of this particular type. Nevertheless, the fact that $46 \%$ of the strains tested were carrying this type of converting phage suggests that a fair proportion of the mitis strains present in the area of origin were carriers of this phage.

A final point with respect to the present findings is whether there is some significance to the observation that the converting phage type isolated from the western strains is related to the $\beta$ - and $\gamma$-phages studied previously. $\beta$-phage was not isolated in Washington and probably was isolated in Ontario or Quebec, Canada, by Toshach from whom Freeman received it (Freeman, 1951). A phage apparently related to $\beta$ has also been isolated by Narbutowicz in Poland (1955). It would thus appear that phages of this type are present in widely separated areas of the world. More studies are needed to determine whether there are other converting phage types as determined by the serological criterion or whether this is indeed a special class of phages. Ideally such studies should be carried out with converting phages isolated from all types of Corynebacterium diphtheriae.

This work was supported in part by State of Washington funds for medical and biological research.

\section{REFERENCES}

Christensen, W. B. (1946). Urea decomposition as a means of differentiating Proteus and Paracolon cultures from each other and from Salmonella and Shigella types. J. Bact. 52, 461 .

Christensen, P. E. (1957). Studies on lysogenicity in C. diphtheriae. Acta path. microbiol. scand. 41, 67 .

Diagnostic Procedures and Reagents (1950). Third edition. American Public Health Association Inc., New York, N.Y.

Freeman, V. J. (1951). Studies on the virulence of bacteriophage-infected strains of Corynebacterium diphtheriae. J. Bact. 61, 675.

Groman, N. B. \& Eaton, M. (1955). Genetic factors in Corynebacterium diphtheriae conversion. J. Bact. 70, 637.

Hewitr, L. F. (1952). Diphtheria bacteriophages and their relation to the development of bacterial variants. J. gen. Microbiol. 7,362 .

HewitT, L. F. (1954). Mechanism of virulence transfer by bacterial viruses. J. gen. Microbiol. 11, 272.

Keogh, E. V., Simmons, R. T. \& Anderson, G. (1938). Type specific bacteriophages for Corynebacterium diphtheriae. J. Path. Bact. 46, 565.

Minzel, G. H. \& Freeman, V. J. (1950). Serologic studies of Corynebacterium diphtheriae. I. The use of a surface active agent in the preparation of uniform suspensions of $C$. diphtheriae for serologic typing. Amer. J. publ. Hlth, 51, 300.

Mueller, J. H. \& Miller, P. A. (1946). A new tellurite plating medium and some comments on the laboratory diagnosis of diphtheria. J. Bact. 51, 743.

Narbutowicz, B. (1955). Studies on the conversion to toxigenicity of phageinfected atoxic strains of Corynebacterium diphtheriae. Acta microbiol. polonica, 4, 245 . 
Parsons, E. I. (1955). Induction of toxigenicity in non-toxigenic strains of $C$. diphtheriae with bacteriophages derived from non-toxigenic strains. Proc. Soc. exp. Biol., N.Y. $90,91$.

Thibaut, J. \& FrédéricQ, P. (1952). Liberation de bactériophage par des souches lysogènes de $C$. diphtheriae sous l'effet des rayons ultraviolets. C.R. Soc. Biol., Paris, 146, 1627.

Thibaut, J. \& Frédéricq, P. (1956). Recherches sur la classification des souches de Corynebacterium diphtheriae type gravis d'après leur sensibilité à divers bactériophages. C.R. Soc. Biol., Paris, 150, 1039.

(Received 8 July 1958) 